\title{
Peer Group Filter for Impulsive Noise Removal in Color Images
}

\author{
Bogdan Smolka \\ Silesian University of Technology, Department of Automatic Control, \\ Akademicka 16 Str, 44-100 Gliwice, Poland \\ smolka@ieee.org
}

\begin{abstract}
In this paper a new approach to the problem of impulsive noise removal in color images is presented. The proposed method is based on the evaluation of the statistical properties of a sorted sequence of the accumulated distances used for the calculation of the vector median of samples belonging to the filtering window. The statistical analysis is performed using the Fisher's linear discriminant, which enables the detection of outliers introduced by the noise process. The described filter output switches between the vector median and the original central pixel. In order to increase the filter's performance, two thresholds are introduced, which enhance the detail preserving abilities of the proposed filtering scheme. The described filtering technique is robust, fast and therefore it can be used in real time denoising applications.
\end{abstract}

Keywords: Color image processing, noise reduction, image enhancement.

\section{Introduction}

The most popular family of nonlinear filters for impulsive noise removal in color images is based on the order statistics [1]. These filters perform the ordering of a set of vectors from the filtering window in order to determine the output sample.

Let the color image $\boldsymbol{x}$ be defined as a mapping $Z^{2} \rightarrow Z^{3}$ and let $W=\left\{\boldsymbol{x}_{k} \in\right.$ $\left.Z^{2} ; k=1,2, \ldots, n\right\}$ represents a square filtering window consisting of $n$ color pixels centered at pixel $\boldsymbol{x}_{1}$. In the case of color image filtering, the most popular denoising approaches are based on a vector ordering scheme defined through the ordering of aggregated distances. The aggregated distance $r_{k}$ assigned to a sample $\mathbf{x}_{k}$, for $k=1,2, \ldots, n$ is defined as

$$
r_{k}=\sum_{j=1}^{n}\left\|\boldsymbol{x}_{k}-\boldsymbol{x}_{j}\right\|_{l}=\sum_{j=1}^{n}\left(\sum_{q=1}^{3}\left|x_{k q}-x_{j q}\right|^{l}\right)^{1 / l}, \quad k=1,2, \ldots, n,
$$

where $\left\|\boldsymbol{x}_{k}-\boldsymbol{x}_{j}\right\|_{l}$ quantifies the distance among two 3-channel samples using the Minkowski metric, where $x_{k q}$ is the $q$-th element of $\mathbf{x}_{k}$ and $l$ characterizes the used norm.

The scalar quantities $r_{1}, r_{2}, \ldots, r_{n}$ can then be sorted in the order of their values and the associated vectors can be correspondingly ordered [2,1]

$$
r_{(1)} \leq r_{(2)} \leq \ldots \leq r_{(n)} \quad \Rightarrow \quad \boldsymbol{x}_{(1)} \leq \boldsymbol{x}_{(2)} \leq \ldots \leq \boldsymbol{x}_{n},
$$

J. Ruiz-Shulcloper and W.G. Kropatsch (Eds.): CIARP 2008, LNCS 5197, pp. 699707, 2008.

(C) Springer-Verlag Berlin Heidelberg 2008 
where $r_{(1)}$ and $r_{(n)}$ is the smallest and the largest accumulated distance. The vector $\boldsymbol{x}_{(1)}$ associated with $r_{(1)}$ is called the vector median, as this vector minimizes the sum of distances to all other pixels contained in $W$ [3].

Nonlinear, ranked type multichannel filters generally define the vector $\mathbf{x}_{(1)}$ as the output of the filtering operation. This selection is due to the fact that vectors that diverge greatly from the data population usually appear in higher indexed locations in the ordered sequence (2).

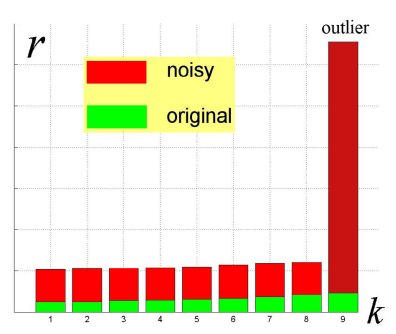

(a)

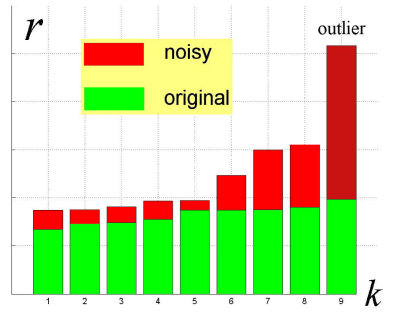

(b)

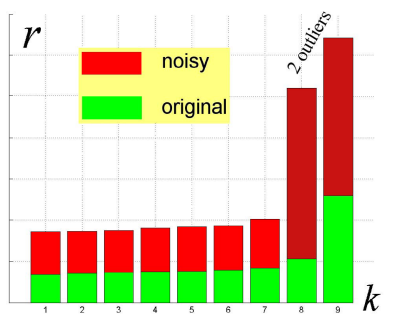

(c)

Fig. 1. Plots of the ordered sequence of the aggregated distances $r_{k}$ assigned to pixels from a $3 \times 3$ filtering window in the original and noisy image. In cases (a) and (b) the filtering window of the noisy image contains one corrupted pixel, whereas plot (c) depicts the situation in which two pixels were contaminated.

Figure 1 depicts the plot showing the sorted sequence of the accumulated distances for pixels in a filtering window of the original and noisy test image. Figures $1 \mathrm{k}$ ) and b) show the distribution of a set of pixels affected by one impulse and Fig. 1:) depicts the situation, in which two pixels in the filtering window were disturbed by the impulsive noise process. Analyzing the sorted sequence of the $r_{k}$ values, it is relatively easy to discriminate between the pixels of the original, undisturbed image and the impulses injected into the image by the noise. This observation leads to the conclusion that the distribution of the sorted aggregated distances can be used for the detection of pixels corrupted by the noise process.

For the discrimination between the uncorrupted and noisy pixels, the aggregated distances can be divided in two sets. One set of the $r$ values will be associated with the "clean" pixels and the second set will consist of the impulses injected into the image by the noise process. It is possible to apply some adaptive thresholding technique to detect the impulses when considering the $r_{k}$ values, however much more efficient is the use of a simple technique, which enables robust and reliable detection of outliers.

For the task of grouping the pixels into two sets of the undisturbed and noisy pixels, the Fisher's linear discriminant can be used. Fisher's linear discriminant is a classification method that generally projects high-dimensional data onto a line and performs classification in this one-dimensional space. In our case, the dimensionality reduction of the color vectors, is performed by the calculation of the aggregated distances.

The goal of the discriminant is to maximize the distance between the means of two classes, while minimizing the variance within each class. This defines the Fisher 


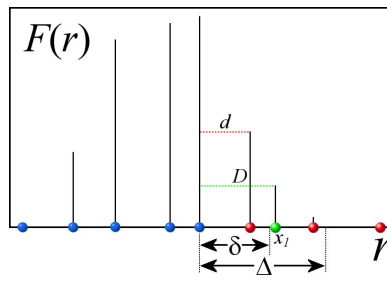

(a)

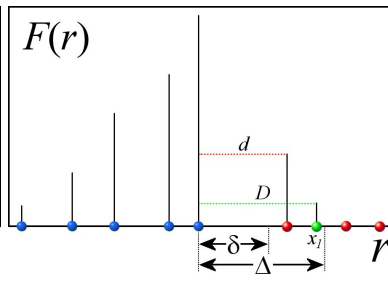

(b)

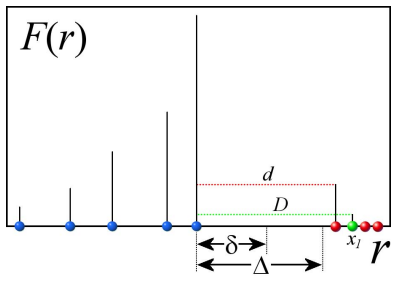

(c)

Fig. 2. Illustration of the thresholding techniques applied to the values of Fishers's discriminant working on the aggregated distances $r$ assigned to pixels from a filtering window. Blue dots represent the pixels declared to be noise-free, green dot denotes the aggregated distance of the central pixel $\boldsymbol{x}_{1}$ and the remaining pixels are marked red.

criterion function $F(k)$, where $k$ denotes the rank of the sorted sequence of the sorted $r_{(k)}$ values

$$
F(k)=\frac{\left[m_{1}(k)-m_{2}(k)\right]^{2}}{v_{1}(k)+v_{2}(k)}, k=1, \ldots, n-1,
$$

where

$$
\begin{gathered}
m_{1}(k)=\frac{1}{k} \sum_{i=1}^{k} r_{(i)}, \quad m_{2}(k)=\frac{1}{n-k} \sum_{i=k+1}^{n} r_{(i)}, \\
v_{1}(k)=\sum_{i=1}^{k}\left(r_{(i)}-m_{1}\right)^{2}, v_{2}(k)=\sum_{i=k+1}^{n}\left(r_{(i)}-m_{2}\right)^{2}, k=1, \ldots, n-1 .
\end{gathered}
$$

The values $m_{1}$ and $m_{2}$ denote the mean values of the two classes of pixels and $v_{1}$ and $v_{2}$ stand for the variances of the aggregated distances.

The classification of the pixels belonging to a filtering window is illustrated in Fig.2 The test sequence of aggregated distances is composed of the values originating from the clean and noisy pixels in $W$. Each of the plots shown in Fig. 2 shows a different distribution of the pixels in the filtering window.

In each case the, function of the Fisher discriminant (3) attains its maximum at $k=5$, and the first five pixels are classified as clean and the remaining are declared to be noisy. The efficiency of such an approach is quite high in the presence of impulse noise in the filtering window. However, the classification of the pixels into two groups is performed in every filtering window and in a situation in which all the pixels are not affected by noise, the division of the pixels into two groups can lead to erroneous classification of the original, undisturbed pixels. Such a case is depicted in Fig. 2a), which shows a situation in which the Fisher's discriminant declared five pixels to belong to the class of original samples and the remaining four were classified as outliers. If the central pixel in this example has a rank higher than five, then this pixel will be unnecessary replaced by the vector median.

To alleviate this problem two thresholds were introduced. The first threshold $\Delta$ is applied to the difference between the $r_{1}$, which denotes the value of the aggregated distance assigned to the central pixel $\boldsymbol{x}_{1}$ and the $r_{\left(k^{*}\right)},\left(k^{*}=\arg \max F(k)\right)$, which 

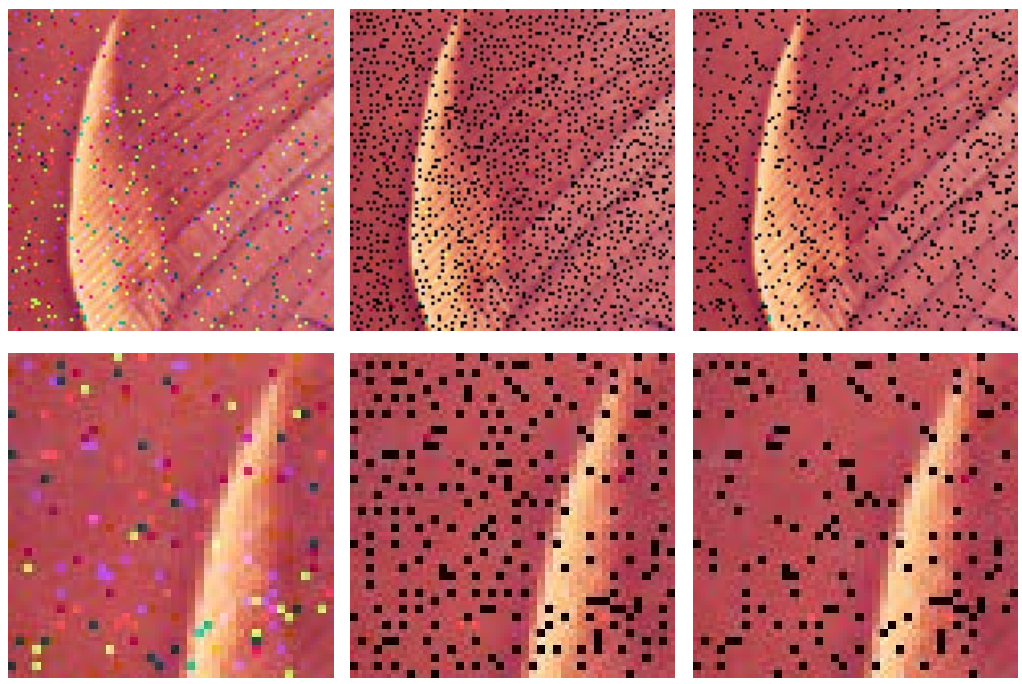

Fig. 3. Illustration of the efficiency of the Fisher's criterion combined with the thresholding technique: (left) part of the noisy test image LENA, (middle) pixels which were detected as impulses using the Fisher's criterion are marked black, (right) after the application of the threshold $\Delta$ the amount of wrongly replaced pixels significantly decreased, $(\Delta=15)$. Below, the zoomed parts of the images are shown.

denotes the aggregated distance maximizing the Fisher's function. In the example provided by Fig. 2 , $k^{*}$ is equal to 5 .

In this way the central pixel $\boldsymbol{x}_{1}$ is defined as an outlier if: $D=r_{1}-r_{k^{*}}>\Delta$. In the example from Fig. 2 in the first case (a), only the pixel with highest rank is declared as an outlier and the pixel $\boldsymbol{x}_{1}$ belongs to the uncorrupted samples. In (b) two pixels are classified as impulses, while the central pixel satisfies the condition $D<\Delta$ and again is classified as not contaminated. In the third plot (c) four pixels are declared to be corrupted, among them the central pixel, as in this case $D>\Delta$.

It can be observed that after the application of the threshold $\Delta$ the amount of pixels detected as corrupted and replaced by the vector median is drastically decreasing as shown in Fig. 3. After the introduction of the $\Delta$ threshold, the pixels with low values of $r$ are being classified as not corrupted and are preserved by the extended algorithm.

However using the $\Delta$ threshold, there are cases like in Fig. 2 a) and b), where the pixels declared as outliers can in fact belong to the set of uncorrupted pixels. To further diminish the classification error a second threshold denoted as $\delta$ is introduced.

The difference between $r_{\left(k^{*}+1\right)}$ and $r_{\left(k^{*}\right)}$ can be used as a measure of the homogeneity of the distribution of the $r_{k}$ values. If the value: $d=r_{\left(k^{*}+1\right)}-r_{\left(k^{*}\right)}$ is small, then it means that most probably the set of samples does not contain any outliers.

In the examples provided by Fig. (2h) the value of $d$ is smaller than the threshold $\delta$ and therefore all the pixels are treated as uncorrupted by noise. In Fig. 2b) $d$ is larger than the threshold $\delta$ and therefore the final classification is performed with regard to the threshold $\Delta$. In the last example (Fig. 2 k) the four pixels with the highest ranks are classified as impulses, as $d>\delta$ and $D>\Delta$. 


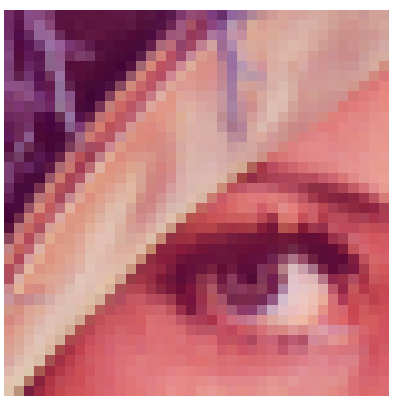

(a)

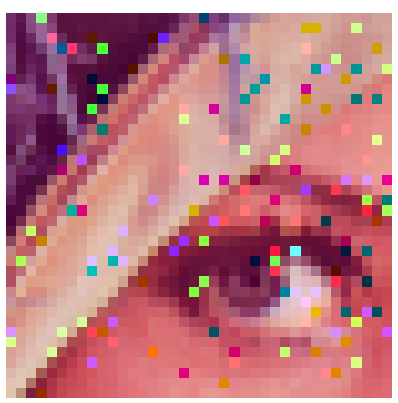

(b)

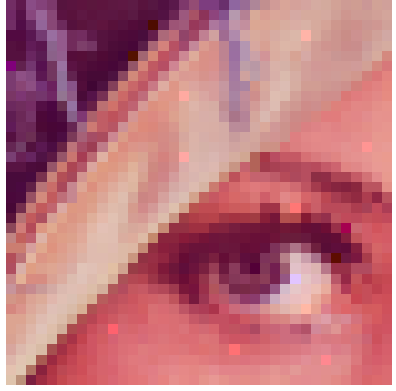

(c)

Fig. 4. Illustration of the efficiency of the proposed filtering technique: (a) test image LENA, (b) image contaminated by impulse noise $(p=0.1)$, (c) output of the proposed filter with two optimal thresholds $\Delta$ and $\delta$

The rule for the replacement of the corrupted central pixel $\boldsymbol{x}_{1}$ by the vector median is then

$$
\boldsymbol{y}_{1}= \begin{cases}\boldsymbol{x}_{(1)}, & \text { if } d>\delta \text { and } D>\Delta, \\ \boldsymbol{x}_{1}, & \text { otherwise, }\end{cases}
$$

where $\boldsymbol{y}_{1}$ is the filter output and $\boldsymbol{x}_{(1)}$ is the vector median of the samples in $W$. In this way the central pixel is classified as noisy if both thresholds are exceeded.

\section{Filtering Performance}

In this work the noisy image pixels are modeled as $\boldsymbol{x}_{i}=\left\{x_{i 1}, x_{i 2}, x_{i 3}\right\}$, where $x_{i q}=$ $\rho_{i q}$ with probability $\pi$ and $o_{i q}$ with probability $1-\pi$, where $\boldsymbol{o}_{i q}$ denotes the $q$-th component of the original pixel at a position $i$ and and the contamination component $\rho_{i q}$ is a random variable in which the variable $\rho$ takes any discrete value in the range $[0,255]$. The contamination of the color image components is uncorrelated, and the overall distorsion rate is $p=1-(1-\pi)^{3}$.

For the measurement of the restoration quality, the commonly used Root Mean Squared Error (RMSE) expressed through the Peak Signal to Noise Ratio (PSNR) was used, as the RMSE is a good measure of the efficiency of impulsive noise suppression. For the evaluation of the detail preservation capabilities the Mean Absolute Error (MAE) has been applied. Additionally, since RGB is not a perceptually uniform space, the restoration errors were also analyzed using the Normalized Color Difference (NCD) [1]

The subjective evaluation of the proposed filter's performance is provided by Fig. 4 As can be seen the filter preserves very well the image details and effectively replaces the impulse noise. Figure 5 shows the dependence of the PSNR on the two thresholds $\Delta$ and $\delta$ using the LENA color test image contaminated by impulsive noise. As can be observed, for contamination rates ranging from $5 \%$ to $20 \%$ the peak value of PSNR is obtained for $\Delta \approx 15$ and $\delta \approx 10$. The same values of the thresholds yield optimal results in terms of PSNR for other color images as depicted in Figs. 6 and 7 which 
Table 1. Comparison of the new filter with the state of the art denoising techniques using the GOLDHILL test image contaminated with impulse noise, $p=10 \%$

\begin{tabular}{|c||c|c|c|}
\hline FILTER & MAE & PSNR & NCD \\
\hline NONE & 4.40 & 19.94 & 99.15 \\
PROPOSED & $\mathbf{0 . 7 9}$ & $\mathbf{3 5 . 8 3}$ & $\mathbf{7 . 9 6}$ \\
Peer Group Filter, [4] & 1.01 & 31.14 & 22.51 \\
Fast Peer Group Filter, [5] & 0.81 & 35.18 & 8.91 \\
Peer Region Determination Filter, [6] & 0.86 & 34.80 & 8.27 \\
Iterative Peer Group Switching Vector Filter, [7] & 1.02 & 31.52 & 21.16 \\
Vector Median Filter, [3] & 4.84 & 30.11 & 43.23 \\
Basic Vector Directional Filter, [1] & 6.68 & 27.27 & 47.67 \\
Directional Distance Filter, [1] & 5.00 & 29.81 & 42.62 \\
Vector Directional Order-Statistics Filter, [8] & 0.91 & 34.62 & 7.63 \\
Hybrid Directional Filter, [1] & 4.94 & 30.27 & 43.46 \\
Local Information Measure Filter, [9] & 3.41 & 31.29 & 30.83 \\
Vector LUM Smoother, [10] & 0.79 & 35.78 & 8.18 \\
Adaptive Vector LUM Smoother, [11] & 1.39 & 34.58 & 10.94 \\
Modified ICM Method, [12] & 0.73 & 35.96 & 8.23 \\
Sigma Filter, [13] & 2.94 & 31.74 & 25.94 \\
Adaptive Video Filtering Framework, [14] & 0.66 & 36.90 & 6.27 \\
Adaptive Vector Median Filter, [15] & 0.95 & 34.10 & 12.17 \\
Adaptive Color Image Filter, [16] & 0.92 & 35.07 & 7.29 \\
Central Weighted Vector Median Filter, [17] & 1.69 & 33.97 & 14.55 \\
Modified Central Weighted Vector Median Filter, [17] & 1.05 & 34.58 & 10.11 \\
Fast Adaptive Noise Reduction Filter, [18] & 0.81 & 35.54 & 8.59 \\
Self-Adaptive Noise Reduction Filter, [19] & 0.79 & 35.71 & 8.46 \\
Generalized Adaptive Vector Sigma Filters, [20] & 1.31 & 34.29 & 12.20 \\
Students t-Test Vector Median Filter, [21] & 1.21 & 35.72 & 15.38 \\
Fast Impulsive Vector Filter, [22] & 1.03 & 34.18 & 8.48 \\
\hline
\end{tabular}

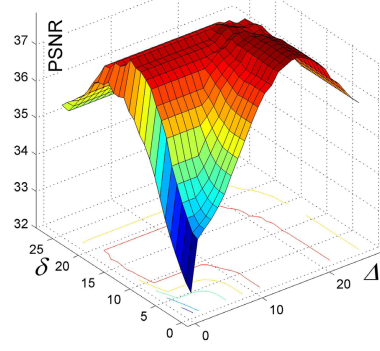

(a) $p=0.05$

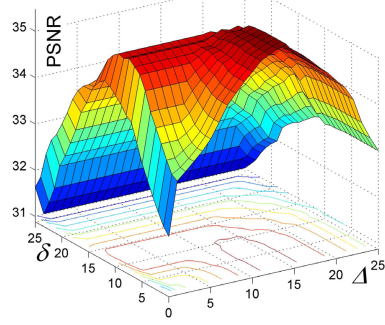

(b) $p=0.1$

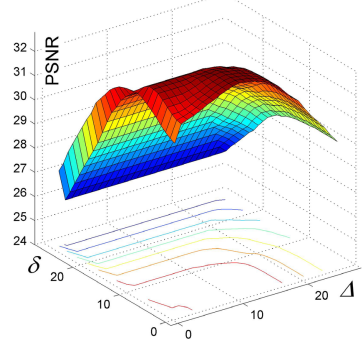

(c) $p=0.2$

Fig. 5. Dependence of the PSNR quality measure on the values of the $\Delta$ and $\delta$ thresholds for the LENA image contaminated by the impulse noise 


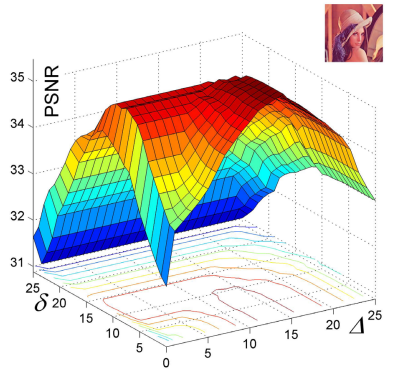

(a) LENA, $p=0.1$

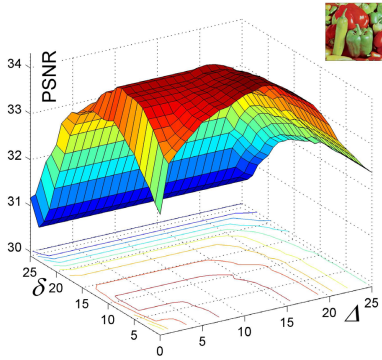

(b) PEPPERS, $p=0.1$

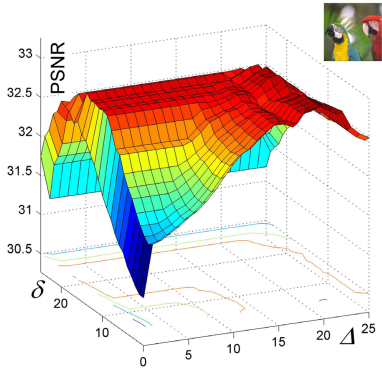

(c) PARROTS, $p=0.1$

Fig. 6. Dependence of the PSNR on the values of thresholds $\Delta$ and $\delta$ for test images LENA, PEPPERS and PARROTS contaminated with impulse noise of $p=0.1$

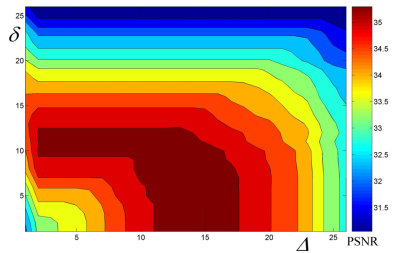

(a) LENA

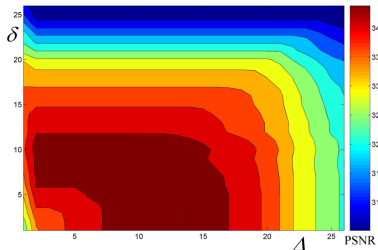

(b) PEPPERS

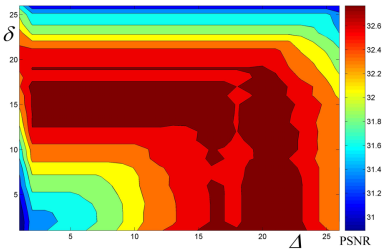

(c) PARROTS

Fig. 7. Surface plots of the dependence of the PSNR on the value of $\Delta$ and $\delta$ for the noisy test images: LENA, PEPPERS and PARROTS, $(p=0.1)$

present the comparison of the dependence of PSNR on the two thresholds for the LENA, PEPPERS and PARROTS color test images polluted by $10 \%$ impulse noise. The lack of dependence of the optimal settings of the two thresholds is extremely beneficial as various images contaminated with unknown amount of impulse noise can be restored using the default thresholds setting.

Table 1 summarizes the restoration quality values obtained when filtering the color test image GOLDHILL contaminated with $10 \%$ impulse noise using the thresholds values equal to: $\Delta=15$ and $\delta=10$. The comparison shows that the proposed technique, despite its simplicity excels over many much more sophisticated filtering designs and is comparable with the best filters known from the literature.

\section{Conclusions}

In the paper a new filtering approach intended for the removal of impulsive noise in color images has been presented. The structure of the new filter is based on the analysis of the Fisher's linear discriminant, which can efficiently discriminate between the original image pixels and impulses introduced into the image by impulsive noise process. The results show that the performance of the proposed filter is very high and is 
comparable with the best known restoration methods. The proposed filter is comparable in speed with the standard VMF, is easy to implement and can be used in applications in which the prefiltering of impulse noise is required.

\section{References}

1. Plataniotis, K.N., Venetsanopoulos, A.N.: Color Image Processing and Applications. Springer, Heidelberg (2000)

2. Pitas, I., Tsakalides, P.: Multivariate Ordering in Color Image Processing. IEEE Trans. on Circuits and Systems for Video Technology 1(3), 247-256 (1991)

3. Astola, J., Haavisto, P., Neuvo, Y.: Vector Median Filters. Proc. of the IEEE 78(4), 678-689 (1990)

4. Kenney, C., Deng, Y., Manjunath, B.S., Hewer, G.: Peer Group Image Enhancement. IEEE Trans. on Image Processing 10(2), 326-334 (2001)

5. Smolka, B., Chydzinski, A.: Fast Detection and Impulsive Noise Removal in Color Images. Real-Time Imaging 11(5-6), 389-402 (2005)

6. Ho, J.Y.F.: Peer Region Determination Based Impulsive Noise Detection. In: Proceedings of ICASSP, Hong Kong, vol. 3, pp. 713-716 (2003)

7. Morillas, S., Gregori, V., Peris-Fajarnés, G.: Isolating Impulsive Noise Pixels in Color Images by Peer Group Techniques. Computer Vision and Image Understanding 110(1), 102-116 (2007)

8. Lukac, R.: Color Image Filtering by Vector Directional Order-Statistics. Pattern Recognition and Image Analysis 12(3), 279-285 (2002)

9. Beghdadi, A., Khellaf, K.: A Noise-Filtering Method Using a Local Information Measure. IEEE Trans. on Image Processing 6(6), 879-882 (1997)

10. Lukac, R.: Vector LUM Smoothers as Impulse Detector for Color Images. In: Proceedings of European Conference on Circuit Theory and Design (ECCTD), Espoo, Finland, vol. 3, pp. 137-140 (2001)

11. Lukac, R., Marchevsky, S.: Adaptive Vector LUM Smoother. In: Proceedings of ICIP, vol. 1, pp. 878-881. Thessaloniki, Greece (2001)

12. Park, J., Kurz, L.: Image Enhancement Using the Modified ICM Method. IEEE Trans. on Image Processing 5(5), 765-771 (1996)

13. Lee, J.S.: Digital Image Smoothing and the Sigma Filter. Computer Vision, Graphics, and Image Processing 24(2), 255-269 (1983)

14. Lukac, R., Fischer, V., Motyl, G., Drutarovsky, M.: Adaptive Video Filtering Framework. International Journal of Imaging Systems and Technology 14(6), 223-237 (2004)

15. Lukac, R.: Adaptive Vector Median Filtering. Pattern Recognition Letters 24(12), 1889-1899 (2003)

16. Lukac, R.: Adaptive Color Image Filtering Based on Center-Weighted Vector Directional Filters. Multidimensional Systems and Signal Processing 15(2), 169-196 (2004)

17. Smolka, B.: Efficient Modification of the Central Weighted Vector Median Filter. In: Van Gool, L. (ed.) DAGM 2002. LNCS, vol. 2449, pp. 166-173. Springer, Heidelberg (2002)

18. Smolka, B., Lukac, R., Chydzinski, A., Plataniotis, K.N., Wojciechowski, K.: Fast Adaptive Similarity Based Impulsive Noise Reduction Filter. Real-Time Imaging 9(4), 261-276 (2003)

19. Smolka, B., Plataniotis, K.N., Chydzinski, A., Szczepanski, M., Venetsanopulos, A.N., Wojciechowski, K.: Self-adaptive Algorithm of Impulsive Noise Reduction in Color Images. Pattern Recognition 35(8), 1771-1784 (2002) 
20. Lukac, R., Smolka, B., Plataniotis, K.N., Venetsanopoulos, A.N.: Generalized Adaptive Vector Sigma Filters. In: Proceedings of IEEE International Conference on Multimedia and Expo (ICME), pp. 537-540. Baltimore, USA (2003)

21. Camacho, J., Morillas, S., Latorre, P.: Efficient Impulsive Noise Suppression Based on Statistical Confidence Limits. J. of Imaging Science and Technology 50(5), 427-436 (2006)

22. Morillas, S., Gregori, V., Peris-Fajarne's, G., Latorre, P.: A Fast Impulsive Noise Color Image Filter Using Fuzzy Metrics. Real-Time Imaging 11(5-6), 417-428 (2005) 